\title{
Electrocoagulación en el agua de proceso de una Industria de alimentos
}

\section{Electrocoagulation in the process water of a food Industry}

\author{
MARTIN DEL CAMPO-SANCHEZ, María Guadalupe †*, ESCOBAR-GONZALEZ, José Luis y \\ PEDROZA-BENITEZ, Socorro
}

Universidad Tecnológica del Valle de Toluca. Carrera de Tecnología Ambiental

ID $1^{\mathrm{er}}$ Autor: María Guadalupe, Martin del Campo-Sanchez / ORC ID: 0000-0003-2689-1684

ID $1^{\mathrm{er}}$ Coautor: José Luis, Escobar-Gonzalez / ORC ID: 0000-0002-932-928X

ID $2^{\text {do }}$ Coautor: Socorro, Pedroza-Benitez / ORC ID: 0000-0002-2745-6994

DOI: $10.35429 / J C P E .2019 .18 .6 .48 .52$

Recibido 09 Febrero, 2019; Aceptado 28 Marzo, 2019

\begin{abstract}
Resumen
El objetivo principal fue desarrollar un sistema de tratamiento de aguas residuales mediante el método de electrocoagulación conforme a los parámetros de Demanda Química de Oxígeno (DQO) y Sólidos Suspendidos Totales (SST). Los resultados obtenidos fueron que a un $\mathrm{pH}$ de 6 y 7 con un tiempo de contacto de 30 minutos se obtuvo el mayor porcentaje de remoción de SST (94.9\%) y DQO (91\%). Una vez seleccionado el conductor se realizaron pruebas a diferentes $\mathrm{pH}(6,7$ y 8$)$ con tiempos de contacto de 15,30 y 45 minutos cada uno, a $12 \mathrm{~V}$ y $0.4 \mathrm{~A}$. Se realizó una prueba estadística ANOVA Multifactorial para determinar si existían diferencias significativas entre tratamientos. Una vez evaluados los resultados se diseñó un prototipo de aproximadamente $38 \mathrm{~L}$ con placas paralelas con resultados de remoción de SST del $93 \%$ y DQO del 66\%. El costo aproximado del tratamiento por metro cúbico es de $\$ 35$.
\end{abstract}

Electrocoagulación, DQO, Prototipo

\begin{abstract}
The main objective was to develop a wastewater treatment system by means of the electrocoagulation method according to the parameters of Chemical Oxygen Demand (COD) and Total Suspended Solids (SST). The results obtained were that at a pH of 6 and 7 with a contact time of 30 minutes, the highest percentage of removal of SST (98\%) and COD (90\%) was obtained. Once the conductor was selected, tests were carried out at different $\mathrm{pH}(6,7$ and 8) with contact times of 15,30 and 45 minutes each, at 12 $\mathrm{V}$ and 0.4 A. A Multifactorial ANOVA statistical test was performed to determine if there were significant differences between the treatments. Once the results were evaluated, a prototype of approximately $38 \mathrm{~L}$ was designed with parallel plates with results of removal of SST of $93 \%$ and COD of $66 \%$. The approximate cost of treatment per cubic meter is $\$ 35$.
\end{abstract}

\section{Electrocoagulation, COD, Prototype}

\footnotetext{
* Correspondencia al Autor (Correo electrónico: maria.martindelcampo@utvtol.edu.mx)

$\dagger$ Investigador contribuyendo como primer Autor
} 


\section{Introducción}

En la sociedad actual, un gran número de compuestos químicos y contaminantes son descargados al ambiente mediante aguas residuales, muchos de ellos reciben un tratamiento previo ya sea físico, químico o biológico y son degradados fácilmente, sin embargo, existen algunos compuestos denominados refractarios difíciles de eliminar como; colorantes, grasas, aceites, compuestos aromáticos, plaguicidas, los cuales no solo requieren un tratamiento convencional, sino el desarrollo de métodos especiales para removerlos (Ojeda et al., 2012).

El impacto de los vertidos industriales puede ser evaluado por parámetros de medición tales como demanda bioquímica de oxígeno $(\mathrm{DBO})_{5}$ y la demanda química de oxígeno (DQO), mismos que evalúan contenidos de sustancias orgánicas e inorgánicas. Entre los contaminantes que más problemas causan en el tratamiento de aguas residuales, son las grasas y aceites, que son altamente estables, inmiscibles con el agua, proceden de desperdicios alimentarios en su mayoría, a excepción de los aceites minerales que proceden de otras actividades.

Al ser inmiscibles con el agua, van a permanecer en la superficie dando lugar a la aparición de natas y espumas. Estas natas entorpecen cualquier tipo de tratamiento, biológico o físicoquímico, por lo que es recomendable eliminarlos en los primeros pasos del tratamiento de las aguas residuales. En este trabajo se proponen alternativas para incrementar la extracción de grasas, utilizando tratamientos con coagulantes y floculantes.

La electrocoagulación es una técnica utilizada para el tratamiento de las aguas residuales, es un proceso en el cual son desestabilizadas las partículas de contaminantes que se encuentran suspendidas, emulsionadas o disueltas en un medio acuoso, induciendo corriente eléctrica en el agua a través de placas metálicas paralelas de diversos materiales, siendo el hierro y el aluminio los más utilizados (Arango, 2012).

Los factores que pueden influir en el tratamiento de la electrocoagulación son: densidad de corriente, temperatura, $\mathrm{pH}$, conductividad eléctrica, corriente eléctrica.

\section{Metodología}

Para la realización de este trabajo se recolectaron 18 muestras simples de agua residual. Se realizaron los análisis de laboratorio de los siguientes parámetros: Demanda Química de Oxígeno (DQO), Sólidos Suspendidos Totales (SST), Conductividad Eléctrica y $\mathrm{pH}$, siguiendo los procedimientos de las Normas Oficiales Mexicanas correspondientes.

Los Sólidos Suspendidos Totales se analizaron con el equipo "Colorimeter Handbook DR/890. Tomando una muestra representativa de $20 \mathrm{ml}$, a los 3 diferentes tiempos (15,30 y 45 minutos), se llevó a cabo de la misma manera diluciones de 20:4 debido a la alta concentración inicial, también se analizó un blanco.

La conductividad eléctrica se analizó con un equipo Pocket-size/EC/TD/Temp/EC59 tomando una muestra representativa, llevando a cabo dilución 10:4 para poder medir dicha concentración.

El pH se analizó con un medidor multiparamétrico de $\mathrm{pH}$ (Thermo Scientific VSTAR40A), tomando como referencia $\mathrm{pH}$ de 6,7 y 8 , ya que el $\mathrm{pH}$ promedio era de 7 , se ajustaba con sosa caustica y ácido sulfúrico según se presentaba el caso.

\section{Selección de material conductor}

Se llevaron a cabo 5 pruebas combinando los conductores (Al y Fe), para conocer cual tenía una mejor remoción en cuanto a SST y DQO, posterior a esto los resultados obtenidos fueron analizados por el programa estadístico Statgraphics.

\section{Tratamientos a diferentes pH y tiempos de contacto}

Así mismo se realizaron pruebas a $\mathrm{pH} 6,7$ y 8 con tiempos de contacto de 15,30 y 45 minutos, siendo un total de 9 ensayos. Los resultados se analizaron mediante un programa estadístico de Statgraphics. 


\section{Diseño de prototipo}

Después de realizar la experimentación en contenedores de uno y dos litros, se diseñó un contenedor de $33.75 \mathrm{~L}$, al cual se le agregaron válvulas de media pulgada a diferentes alturas como puntos de muestreo y salidas de purga.

\section{Distribución de placas}

En el diseño del prototipo se colocan sujetadores para 18 placas distribuidas en 3 filas de 6 placas con una separación de $4.1 \mathrm{~cm}$ entre cada una y $5 \mathrm{~cm}$ de distancia entre cada fila.

\section{Costo beneficio}

Se llevó a cabo un análisis costo beneficio determinado por la energía consumida por volumen de agua tratada en Kw.h/m3.

\section{Resultados}

\section{Material conductor}

En la Tabla 1 se muestran los resultados para la selección de placas, se realizaron pruebas con aluminio y acero inoxidable, con diferentes tipos de combinaciones con un tiempo de contacto de 30 minutos.

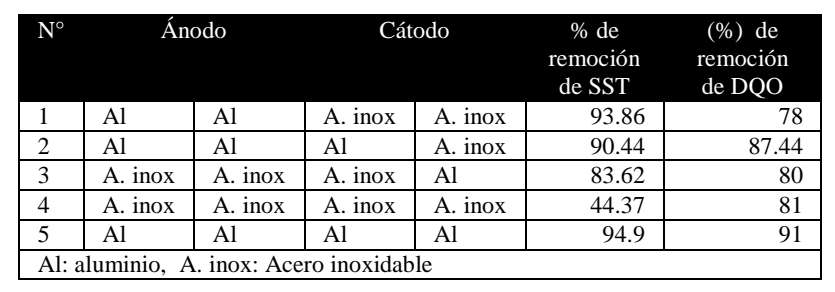

Tabla 1 Resultados de porcentaje (\%) de remoción con diferentes combinaciones de placas

Se realizó un análisis de regresión lineal para ver la relación existente entre la remoción y tipo de electrodo, pues existe una relación estadísticamente significativa $(p<0.05)$ entre aluminio y acero inoxidable, siendo el aluminio el que presenta menor dispersión de datos. Los materiales conductores demuestran estadísticamente que existe diferencia significativa $(p<0.05)$ en el tipo de materiales con respecto al aluminio-acero inoxidable siendo el aluminio el mejor material para remoción de SST y DQO además que se observó que en las pruebas el acero inoxidable generaba turbidez al agua.
Esto podría ser por el tipo de aleación existente en la materia, por que pueda contener Fe.En la Tabla 2. Se presentan los resultados de las concentraciones de SST y DQO en las pruebas realizadas a una concentración inicial de $1,465 \mathrm{mg} / \mathrm{L}$ y $7,800 \mathrm{mg} / \mathrm{L}$ respectivamente, con diferentes $\mathrm{pH}$ y tiempos de contacto.

\begin{tabular}{|c|c|c|c|}
\hline tiempo & pH & SST & DQO \\
\hline 15 & \multirow{3}{*}{6} & 72.3 & 988.7 \\
\hline 30 & & 28.0 & 712.3 \\
\hline 45 & & 13.7 & 731 \\
\hline 15 & \multirow{3}{*}{7} & 74.0 & 981.3 \\
\hline 30 & & 36.3 & 780.0 \\
\hline 45 & & 19.0 & 802.7 \\
\hline 15 & \multirow{3}{*}{8} & 146.0 & 1748.7 \\
\hline 30 & & 120.0 & 1324.7 \\
\hline 45 & & 89.3 & 1242.3 \\
\hline
\end{tabular}

Tabla 2 Concentración de SST y DQO en mg/L a $12 \mathrm{~V}$ y $0.4 \mathrm{~A}$

Los resultados de la Tabla 2 indican que existe una mayor dispersión con respecto a $\mathrm{pH} 8$ en comparación con los $\mathrm{pH}$ de 6 y 7, siendo estos últimos los que mejor remoción de SST a un tiempo de contacto de 45 minutos y DQO a un tiempo de contacto de 30 minutos, además de que estadísticamente no existe diferencia significativa $(p>0.05)$ entre ellos.

El porcentaje de remoción de los SST fue a un tiempo de 45 minutos a pH 6 y 7 con el $99.0 \%$ y $98.7 \%$ respectivamente. Con respecto a la DQO el mayor porcentaje de remoción que se presentó fue a los 30 minutos a pH 6 y 7 con 90.8 $\%$ y $90.0 \%$ respectivamente.

\section{Diseño de prototipo}

En el diseño del prototipo se colocaron sujetadores para 18 placas distribuidas en 3 filas de 6 placas con una separación de $4.1 \mathrm{~cm}$ entre cada una y $5 \mathrm{~cm}$ de distancia entre cada fila.

La separación entre las placas fue a $3 \mathrm{~mm}$ y $5 \mathrm{~mm}$, y se observó un menor desgaste entre cada una a los $3 \mathrm{~mm}$, además que no se tiñen con aguas de color y el material permanece sin presencia de colorante (Figura 1). 

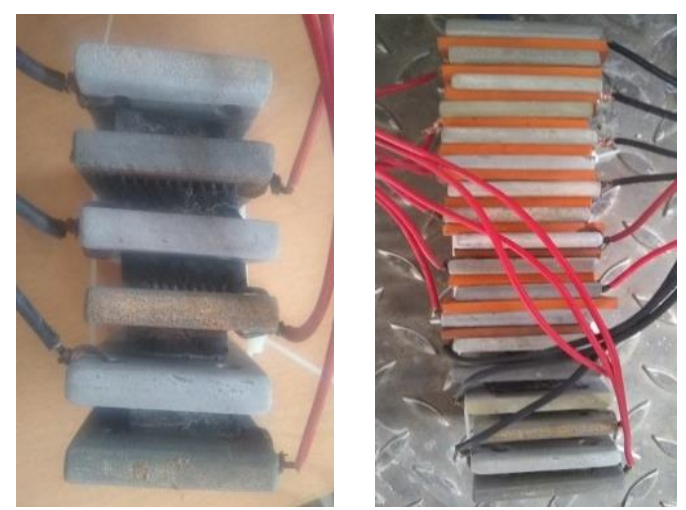

Figura 1 Separación entre placas

A mayor distancia $(5 \mathrm{~mm})$ entre placas éstas presentaron mayor desgaste y en muestras de agua donde se tiene mayor color al final del proceso las placas presentaron impregnación de colorante y grasa. Formándose a su vez mayor lodo en el proceso de remoción.

El lodo presentó un cambio de $\mathrm{pH}$ de 11 llegando hasta 13 a diferencia de cuando las placas presentan menor distancia entre ellas donde se mantiene entre 6 a $7 \mathrm{pH}$.

Se observó que en las pruebas donde las placas tenían mayor separación entre ellas presentaban un olor muy fuerte metálico, contrariamente a menor distancia el olor era menos intenso.

A mayor distancia entre las placas el color en la muestra fue removido significativamente $\quad(p<0.05) \quad$ comparado de cuando estas estaban juntas, por lo que se puede suponer que a mayor distancia se forman más complejos de $\mathrm{Al}(\mathrm{OH})_{3}$ que incrementa la espuma $\mathrm{y}$ el $\mathrm{pH}$.

Por otro lado la remoción de color fue mayor al incrementar la cantidad de placas de aluminio.

En el diseño de prototipo la distribución de placas es un factor importante en la compactación de la cama de lodos.

Se decidió incluir un sistema de micro burbujeo por efecto Venturi con una bomba tipo jet de $1 / 2 \mathrm{HP}$, para que los pequeños flóculos se arrastraran a la superficie y fueran retirados fácilmente (Figura 2).

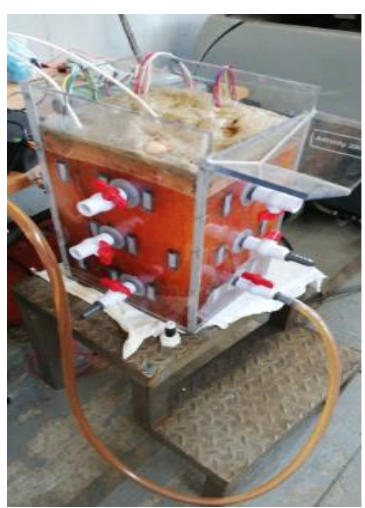

Figura 2 Pruebas realizadas con la microburbuja en el equipo de electrocoagulación

El micro burbujeo generó un menor volumen de lodo bajando entre 3-5\% de la muestra total. Además se observó que la reacción se realizaba normalmente hasta tener una muestra clarificada entre $30-45 \mathrm{~min}$ se redujo a 8-15 min de tiempo de contacto sin afectar la DQO final, además que el lodo se notó más uniforme y espeso reduciéndose de 1-2 L por $33.7 \mathrm{~L}$ en comparación de 3-5 L sin microburbuja.

\section{Costo}

Se estableció el costo energético por metro cúbico de agua tratada, considerando el valor actual de $1 \mathrm{KWh}$ correspondiente a $\$ 1.8558$, dando un total aproximado de $\$ 35.19 / \mathrm{m}^{3}$.

\section{Conclusiones}

El aluminio es el mejor material conductor con un $\mathrm{pH}$ entre 6-7 para el tratamiento de agua con un mayor porcentaje de remoción de SST y DQO con tiempos de contacto de 45 y 30 minutos respectivamente.

La separación entre las placas está directamente relacionada con la cantidad de lodo formada y la impregnación del color y agua del agua de proceso en las placas. El tratamiento con microburbuja presentó menor generación de lodo residual, además de menor tiempo de retención hidráulica.

En el análisis costo beneficio se concluye que el tratamiento por metro cubico es de $\$ 35.19$ por lo que se puede considerar viable. Se recomienda experimentar la viabilidad de trabajar con intensidades de corriente más elevadas para determinar la relación con el tiempo de retención. 


\section{Agradecimiento}

Es importante mencionar nuestro agradecimiento al Ingeniero en Tecnología Ambiental Edgar Margarito Gutierrez por su valiosa participación en este trabajo, pues sin su ayuda no hubiera sido posible la realización de este proyecto.

\section{Conclusiones}

La caracterización de las muestras de agua antes y después del tratamiento permite concluir que el aluminio es el mejor material conductor con un $\mathrm{pH}$ entre 6-7 ya que estadísticamente presenta menor dispersión de datos. El porcentaje de remoción final de DQO fue de $66 \%$ y de $93 \%$ de SST con y sin microburbuja y un tiempo de retención hidráulica de 8-15 minutos.

El tratamiento con microburbuja presenta menor generación de lodo residual, además de menor tiempo de retención hidráulica siendo la opción más viable.

En el análisis costo beneficio se concluye que el tratamiento por metro cubico es de 35.19 MNX por lo que se puede considerar viable.

\section{Referencias}

Arango Ruiz Alvaro. (2012). Efectos del pH y la conductividad en la electrocoagulación de aguas residuales de la industria láctea. Producción más Limpia. 7: 1, pp.59-67. ISSN 1909-0455.

Ojeda Armaignac Elaine, Hing Cortón Romelia y González Díaz Yudith. (2012). Estudio comparativo del uso de electrodos de hierro y aluminio en el proceso de electrocoagulación de la vinaza. CENIC Ciencias Químicas. 43:1. 